\title{
Editorial
}

\section{Managing diabetes: The drivers of change}

\author{
Sanjay Kalra, Ashok Kumar Das ${ }^{1}$, Rakesh Sahay ${ }^{2}$ \\ Department of Endocrinology, Bharti Hospital and BRIDE, Karnal, Haryana, ${ }^{1}$ Department of Medicine, JIPMER, Puducherry, ${ }^{2}$ Department of \\ Endocrinology, Osmania General Hospital, Hyderabad, Andhra Pradesh, India
}

\section{The Challenge}

Diabetes management is a challenging proposition. Recent advances in the etiology and pathophysiology of the disease have changed it from a simple disease of insulin deficiency, to a bifactoral model of insulin deficiency and resistance, to a multifactorial condition. The earlier moniker of the Ominous Octet ${ }^{[1]}$ is now being replaced by the Dirty Dozen $^{[2]}$ as potential candidates for the elusive etiology of type 2 diabetes line up.

The advances in pathophysiology have been accompanied by improvements in pharmacology, and we now have a wide array of anti-diabetic drugs to choose from, These drugs, both oral and injectable, both non-insulin and insulin, can be prescribed in multiple combinations and permutations, separately as well as in fixed dose combinations.

While it may be a challenge for the endocrinologist to understand, remember and use these therapies, this is the least of his or her problems.

The diabetes pandemic shows no sign of abating ${ }^{[3]}$ and not only developing nations such as India but developed countries such as $\mathrm{USA}^{[4]}$ also report a shortage of trained endocrinologists and other professionals, who are qualified to tackle the large number of patients needing diabetes care.

In spite of various physiological, biochemical, and therapeutic advances in diabetology control remains poor. Data from all parts of the globe reports poor control of diabetes. The largest observational trial done in diabetes

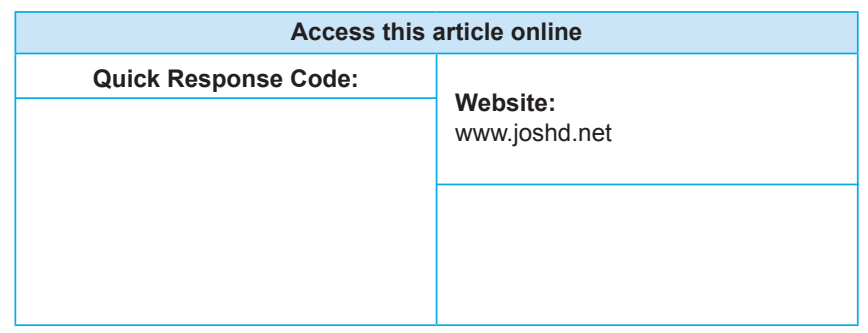

until date, the A1chieve trial, reports an average HbA1c of $9.5 \%$ globally, at the time of insulin initiation. ${ }^{[5]}$ It seems that medical advances alone are not enough to fight the challenge of the diabetes pandemic. Had they been so effective, we would not be reporting the same HbA1c levels worldwide that were reported a decade ago.

\section{LANDMARK ChANgeS In MEDICINE}

Yet, change is not impossible. Mankind has overcome challenges related to disease and sickness in the past.

The history of medicine records landmark changes in our clinical practice. All these changes were fuelled by simple, yet strong, catalysts, which acted as drivers of change. The washing of hands by mid-wives was noted to lead to a reduction in maternal mortality, and introduced asepsis in to operation theaters. Stephane Tarnier noted the use of incubators for new born animals in Paris Zoo, and pioneered their use in new born humans. The high incidence of trauma-related infections during World War II hastened the development of antibiotics. Vaccinations have led to the eradication of smallpox, and hopefully, polio. In all these cases, interested people took charge of a suboptimal situation, and tried to improve it. Change was propelled by a desire to see an improvement in health-care. Change was effected using easily available, economical interventions. The change was measurable within a short period of time, e.g., the reduction in maternal, neonatal or battle field mortality.

With diabetes the situation is not all that different. As diabetes care professionals, we wish to improve the health of our patients. We have multiple drugs and interventions available, and can measure their effect easily. However, we do not seem to be able to take full advantage of them.

\section{CHANGE}

To comfort diabetes effectively, we need change. Apart from change, we also need to change ourselves. 
What is change? The dictionary defines change as the reaction to an existing situation. It may also be called the process of becoming different. Alteration, modification, and transformation are some synonyms of change. ${ }^{[6]}$

A change for the better, or in a positive direction, is what all health-care professionals achieve for when they manage patients with any disorder. This, however, is not so easy to come by.

\section{THE DISCOMFORT OF CHANGE}

Change is often associated with discomfort, ${ }^{[7]}$ whether related to life-style, health-care seeking behavior or treatment, change is challenging for patients, especially those with chronic disease. The person with diabetes needs to change various habits or behavior, some of which may have been ingrained since childhood.

Change is also discomforting for the family, which has to cope with different dietary and physical activity patterns as well as an unwelcome financial impact. The stress associated with a diagnosis of diabetes may devastate not only the patient, but family members as well. Similarly, change is experienced by the community and economy, when people develop diabetes. An increase in work place absenteeism, partly due to appointments at the doctor, and partly to complications of diabetes, negatively impacts the economy, and causes generalized discomfort.

Health-care providers are also not immune to change related to diabetes. Having to shift from an acute care model to a chronic care model, adopt a patient-centered approach, and involve the patient in shared decision making, may be discomforting for the doctor.

For an endocrinologist, the diabetes epidemic means less time and energy spent on classic endocrinopathy: this, too, may be unliked by some.

\section{THE DRIVERS OF CHANGE}

To achieve such a large spectrum of change, we need multiple drivers of change. Perhaps the best drivers will be those who are most affected by the disease. If this were true, the drivers of change in diabetes will turn out to be patients of diabetes, along with their family, community, and governmental decision makers. Diabetes care professionals will also be among the drivers of change in diabetes.

Drivers are people, or objects, who move the reaction of change. A good driver of change will act as a catalyst and ensure positive movement in the fight against diabetes. A good driver will work not only at the clinical or medical level, but also with the socio-cultural, psychological, and epidemiological aspects of the disease.

Diabetes, attitude, wishes, and needs

The widely published diabetes, attitude, wishes, and needs (DAWN) call to action, published in 2004, called for concerted multi-stakeholder action to ensure patient-centered diabetes and chronic care ${ }^{[8]}$ This was based on the finding of the 2001 DAWN study. ${ }^{[9]}$ Developments since then have firmly established the patient centered, chronic care model in diabetology.

As discussed already, however, we needed to involve other stakeholders, too, to drive a change in diabetes. DAWN 2, a multinational study on psychosocial and patient-centered diabetes care, surveyed over 16,000 subjects in 17 countries, covering patients with diabetes, their family members, and health-care professionals. ${ }^{[10]}$ The DAWN 2 study confirms that the physical, financial and emotional burden of diabetes across cultures and countries is carried by the entire family, not just by the person with diabetes. $34 \%$ of family members worldwide report a negative financial impact on themselves due to the diabetes of their loved one, with $93-97 \%$ doing so in India. $20 \%$ of family members from all countries experience that their loved one is being discriminated against because of diabetes and that the community they live in is intolerant of diabetes, while $14-32 \%$ feel the same in India. The results will serve as a bench mark for currently unmet needs and requirements of people with diabetes and their family members. They will define the change that is required, and stimulate the drivers of change to affect it.

\section{WHOSE RESPONSIBILITY?}

But who should bell the cat? As endocrinologists, we have progressed from a conventional, physician-centered model of health-care toward a patient-centered approach in diabetology. ${ }^{[11]}$ As Indian endocrinologists, we have for long been aware of the importance of the community in modifying diabetes care-related behavior of our patients. The most meticulously written prescription and the most intensively delivered health education at times fail in front of anti-insulin or an anti-diabetes care remark made by an influential family member, religious leader, or even neighbor. Thus, we should not limit our interventions and education to patients, but also involve their family and community. The term that we propose for influence of these factors on a patient's diabetes care is "eco-sensitivity" or community-sensitivity. 
Kalra, et al.: Driving change in diabetes

We propose a small step forward in the management of diabetes, from a patient-centered, to a community-oriented approach, especially in oriental cultures which have no stigma attached to the sharing of health-related details.

Within the community, we must identify influential drivers of change, such as religious leaders, community leaders, and office bearers of social or professional groups, More humble professionals such as barbers, hair stylists and restaurant or tea shop owners also make unlikely, yet effective, drivers of change, as they interact with large numbers of people every day. If utilized properly, they can act as "health evangelists" for optimal diabetes care, becoming diabetes change ambassadors in their communities. We support the grooming and training of such ambassadors, who work to sensitize the community to the importance of effective diabetes care.

Religious leaders are a potent, yet underutilized, driver of change for diabetes care. Respected in their communities as opinion leaders, they are looked up to by laity for advice. In many religious societies, the local priest's interpretation of "good health" influences a patient's attitude toward diabetes and diabetes care. We have experienced this in our work with patients, where we successfully use examples of pro-activism from Holy Scriptures and history to motivate them to initiate insulin therapy. ${ }^{[12]}$

Working in a resource-challenged society, we are also acutely aware of the limitations of individuals and non-governmental groups in changing the diabetes scenario. Governmental support is needed at all levels to have a meaningful impact on the diabetes epidemic. If this is obtained, success can be certain, similar to what we have experienced with acute illnesses such as polio and smallpox. If not, we will soon have to refer to diabetes as being endemic in all our countries, instead of calling it an epidemic.

\section{CONCLUSION}

If we are to drive change in diabetes, therefore, we need to involve multiple stakeholders. As responsible diabetes care providers, we should take a lead in sensitizing, educating, and empowering other drivers of change.
Being patient-centric is not enough: we need to actively pursue patient advocacy at all possible platforms including the community, the government and with our peers.

As physicians who are oath bound to take care of our patient's health, we need to take the lead. We need to practice patient advocacy, to ensure that their voice is heard, to involve all stakeholders in driving change through concerted, sustained team work. The onus is upon us.

\section{REFERENCES}

1. Defronzo RA. Banting Lecture. From the triumvirate to the ominous octet: A new paradigm for the treatment of type 2 diabetes mellitus. Diabetes 2009;58:773-95.

2. Kalra S. Recent advances in pathophysiology of diabetes: Beyond the dirty dozen. J Pak Med Assoc 2013;63:277-80.

3. International Diabetes Federation. Diabetes and impaired glucose tolerance. IDF Diabetes Atlas. $5^{\text {th }}$ ed. Brussels, Belgium: International Diabetes Federation; 2011.

4. Stewart AF. The United States endocrinology workforce: A supply-demand mismatch. J Clin Endocrinol Metab 2008;93:1164-6.

5. Home P, Naggar NE, Khamseh M, Gonzalez-Galvez G, Shen C, Chakkarwar P, et al. An observational non-interventional study of people with diabetes beginning or changed to insulin analogue therapy in non-Western countries: The A1chieve study. Diabetes Res Clin Pract 2011;94:352-63.

6. Available from: http://www.en.wiktionary.org/wiki/change. [Last accessed on 2012 May 26].

7. Kalra S, Kumar S, Kalra B, Unnikrishnan A, Agrawal N, Sahay R. Patient-provider interaction in diabetes: Minimizing the discomfort of change. Internet J Fam Pract 2010;8:2.

8. Conference Report: $2^{\text {nd }}$ International DAWN Summit; a call-to-action to improve psychosocial care for people with diabetes. Pract Diabetes Int 2004;21:201-8.

9. Peyrot M, Rubin RR, Lauritzen T, Snoek FJ, Matthews DR, Skovlund SE. Psychosocial problems and barriers to improved diabetes management: Results of the Cross-National Diabetes Attitudes, Wishes and Needs (DAWN) Study. Diabet Med 2005;22:1379-85.

10. Available from: http://www.dawnstudy.com/dawn2/ newsandevents.asp. [Last accessed on 2013 Feb 06].

11. Kalra S, Unnikrishnan AG, Skovlund SE. Patient empowerment in endocrinology. Indian J Endocrinol Metab 2012;16:1-3.

12. Kalra S, Kalra B. Using analogy-building to initiate insulin. Internet J Fam Pract 2010;8:1.

How to cite this article: ???

Source of Support: Nil. Conflict of Interest: None declared. 